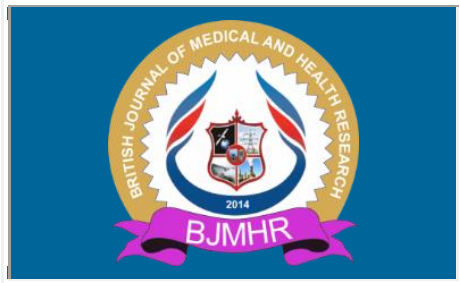

\title{
BJMHR
}

British Journal of Medical and Health Research Journal home page: www.bjmhr.com

\section{Effect of using Polyether Ether Ketone versus Metal Mesh Reinforcement on Fracture Resistance of Maxillary Polymethyl Methacrylate Denture Bases. An In-Vitro study.}

\author{
Ahmed Abd El Gawad Abdelhafeez Hassan Moussa ${ }^{1}$, Amal Rekaby Taha ${ }^{2}$, Fardos \\ Nabil Rizk ${ }^{3}$,Reham B. Osman ${ }^{4,}$ Ahmed Moussa ${ }^{2}$ \\ 1.B.D. S, Faculty of Dentistry, Cairo University, Egypt. Demonstrator Of Oral and \\ Maxillofacial Prosthodontics Department, Faculty of Dentistry, British University, Cairo, \\ Egypt. \\ 2.Professor of Oral and Maxillofacial Prosthodontics Department, Faculty of Dentistry \\ Cairo University Cairo, Egypt. \\ 3.Professor of Oral and Maxillofacial Prosthodontics Department, Faculty of Dentistry, \\ British University, Cairo, Egypt. \\ 4.Prosthodontics Department, Faculty of Dentistry, Cairo University, Cairo, Egypt.
}

\section{ABSTRACT}

The aim of this study was to evaluate the effect of different reinforcing materials on the fracture resistance of maxillary dentures reinforced by either PEEK or metal framework meshes. The weight of reinforced dentures in both groups was also calculated. 14 reinforced complete dentures were fabricated with similar denture base thickness and same arrangement of teeth by duplication of trial denture bases through custom made stone mold and silicon mold for teeth arrangement. The dentures were divided into 2 groups. In the first group, the dentures were reinforced with metal meshes while in other group PEEK meshes of the same thickness was used for the reinforcement. Mesh reinforcement in both groups were digitally designed by EXO-CAD dental software on scanned cast, printed, and then fabricated in either of the two materials: metal or PEEK. Each denture was subjected to load from a universal testing machine using a fissure load applicator at mid palatal area at speed of $5 \mathrm{~mm} / \mathrm{min}$. The load at failure manifested by sharp drop to below $50 \%$ on load-deflection curve was recorded in Newton. Before the load application, the weight of each denture in both groups was evaluated. All collected data were statistically analyzed. Fracture pattern of dentures in both groups was visually assessed. A statistically significant difference $(P=0.004)$ in the maximum average load the dentures could withstand before fracture was found between the two groups. Metal reinforced maxillary dentures could withstand maximum average load of $1686.63 \pm 325.06 \mathrm{~N}$ with corresponding value of $1160.22 \pm 197.21(\mathrm{~N})$ for PEEK reinforced dentures. Also using PEEK mesh decreased weight of denture by $26 \%$ compared to metal reinforced denture. PEEK mesh with thickness of 0.7 to $0.9 \mathrm{~mm}$ did not influence the fracture strength of complete dentures compared to metal mesh with the same thickness.

Keywords: PEEK, reinforcement, maxillary, denture.

*Corresponding Author Email: ahmed.mossa@bue.edu.eg

Received 01 December 2021, Accepted 23 December 2021 


\section{INTRODUCTION}

Various alternatives exist for management of the edentulous predicament. Conventional maxillary complete denture (CD) remains to be one of most accepted and widely used treatment options ${ }^{1}$. Worldwide, polymethyl methacrylate is the most employed denture base material ${ }^{2}$. This can be attributed to its high esthetic properties, ease of manipulation and ease of the repair of the material. On the other hand, the most common encountered complication with PMMA maxillary CDs is midline fracture of prostheses especially in the presence of opposing natural dentition ${ }^{3}$. The reported incidence of fracture of the maxillary CDs is twice that of the mandibular CDs. ${ }^{4}$ Masticatory forces cause increased resorption of edentulous ridges compared to mid-palatal area especially if there is lack of regular prosthodontic maintenance check-ups ${ }^{5}$. Different techniques have been attempted to reinforce CDs by several researchers. At a microscale level, reinforcement is achieved by adding reinforcing powder particles or fibers, and at a larger scale by adding larger-size fibers, wires, metallic or non-metallic meshes ${ }^{4,6}$.

The most common method for the reinforcement of PMMA is the incorporation of fibers or filler particles in the polymer matrix ${ }^{7}$. The matrix forms a continuous phase which surrounds and holds the fibers in place which act as the main load-bearing filler to withstand the force concentration $^{8}$ Silane coupling agent could be used to improve the strength of the adhesion between the matrix and fibers ${ }^{9}$. Among these different reinforcement fibers Nylon fibers, Kevlar fibers, glass fibers, E-glass fibers and PMMA fibers.

Stainless steel wires have been a popular type of metal strengtheners used ${ }^{10}$. A wide range of strengthening effect of metal wires has been reported in the literature, such as effect of various wires form (shape and diameter) on the transverse strength of denture base composite material $^{11}$. it was mentioned that from a technical point of view there was a difficulty in manipulation of metal wires when used as base material strengtheners ${ }^{12}$. The influence of using of a stainless-steel mesh to improve the mechanical properties of acrylic denture base material has been evaluated by several investigators ${ }^{10}$. Furthermore ,from a technical point of view, a fractured denture base previously reinforced with metal mesh would be more difficult to repair because of wires protruding from the fracture site ${ }^{13}$.

Casted metal strengtheners such as plates and meshes have been incorporated in the anterior palate region, to enhance the tensile strength of tested denture bases. The early attempt for denture reinforcement by metal frame and suggested metal framework design for metal based complete maxillary dentures that does not extend to cover the residual ridge crest this design was suggested in order to facilitate arranging the artificial teeth in cases of vertical space limitation $^{14}{ }^{15}$. The benefit of using a rigid metal strengthener is that it increases the fracture 
strength of PMMA in tension side ${ }^{16} 17$ and it was justified in the statement that saying use of cobalt-chromium bases in maxillary dentures reduces the functional bending of the prosthesis 19. The metal-base framework for a complete maxillary denture is designed to mainly cover the palatal and residual ridges areas, with the borders made of acrylic resin. However, it is difficult to arrange the artificial teeth in this design as there is not enough vertical distance between the upper residual ridge crest and the antagonist jaw in case of well-developed ridges $^{18} 19$. Shimizu, $H$. et al showed that the fracture strength of a cobalt-chromium reinforced complete maxillary denture with the newly proposed metal framework design was approximately two times greater than that of non-reinforced resin-based denture ${ }^{20}$. Using metal frameworks within dentures increases the strength of the denture; nevertheless, concerns like corrosion of the metal framework, allergic reactions, permanent deformation following dropping of a denture, and difficulties encountered during casting procedure remain to be problems for metal-supported dentures and encouraged the search for alternative reinforcing materials ${ }^{17}$.

Recently, new and non-metallic reinforcing framework materials have been introduced and suggested as a possible alternative to conventional mesh metal frameworks. Polyether ether ketone (PEEK) is a relatively new biocompatible polymer that has been incorporated in dental field with adequate mechanical properties when used in different restorative approaches $^{21}$.

In current study, the use of PEEK as a reinforcing mesh framework material was evaluated by comparing fracture resistance between PEEK and metal framework reinforced maxillary complete dentures.

\section{MATERIALS AND METHOD}

For fabrication of study models trial denture bases, fourteen stone casts with $8 \mathrm{~mm}$ base thickness were poured using a standard rubber mold of a completely edentulous maxillary model with no undercuts or irregularities (U-402, Nissin Dental Products Inc., Kyoto, Japan). The stone casts were randomly assigned to either of two groups ( $\mathrm{n}=7$ each): metal-reinforced (group A) or PEEK reinforced (group B) maxillary denture bases. On one of the poured casts, 2.5-3 mm thickness trial denture bases of modelling wax, as determined by digital calliper, were softened, and adapted to fabricate trial denture bases. The wax was trimmed as dictated by limiting structures of the maxillary denture except for a small handle like extension to facilitate the removal of fabricated trial bases from casts at a later stage. The cast with wax trial denture base was then flasked. After setting of the stone, the flask was opened, and the modelling wax was thoroughly removed creating a mould for the fabrication of PMMA trial bases. Self-cured PMMA (Acrostone dental manufacture, Cairo, Egypt) was then mixed and 
packed, two members of flask was re-assembled under hydraulic pressure to allow for proper packing of acrylic. After curing of PMMA, flask was opened, and trial denture base was removed and finished. The previous step was repeated till 14 trail denture bases with the same thickness were fabricated.

A maxillary complete denture was then fabricated with the aid of one of the previously made trial denture bases in conventional way using heat cure denture base resin. A new elastomeric mold of the teeth and polished surface of denture was then fabricated. With the aid of fabricated elastomeric mold, fourteen similar trial bases with the same teeth position and trial base thickness as fabricated denture were obtained as shown in (figure 1).

By the end of this stage, all specimens (14-dentures) were fabricated till the pre-flasking step. All denture bases exhibited the same thickness and artificial teeth arrangement and were ready for flasking and reinforcing mesh insertion.

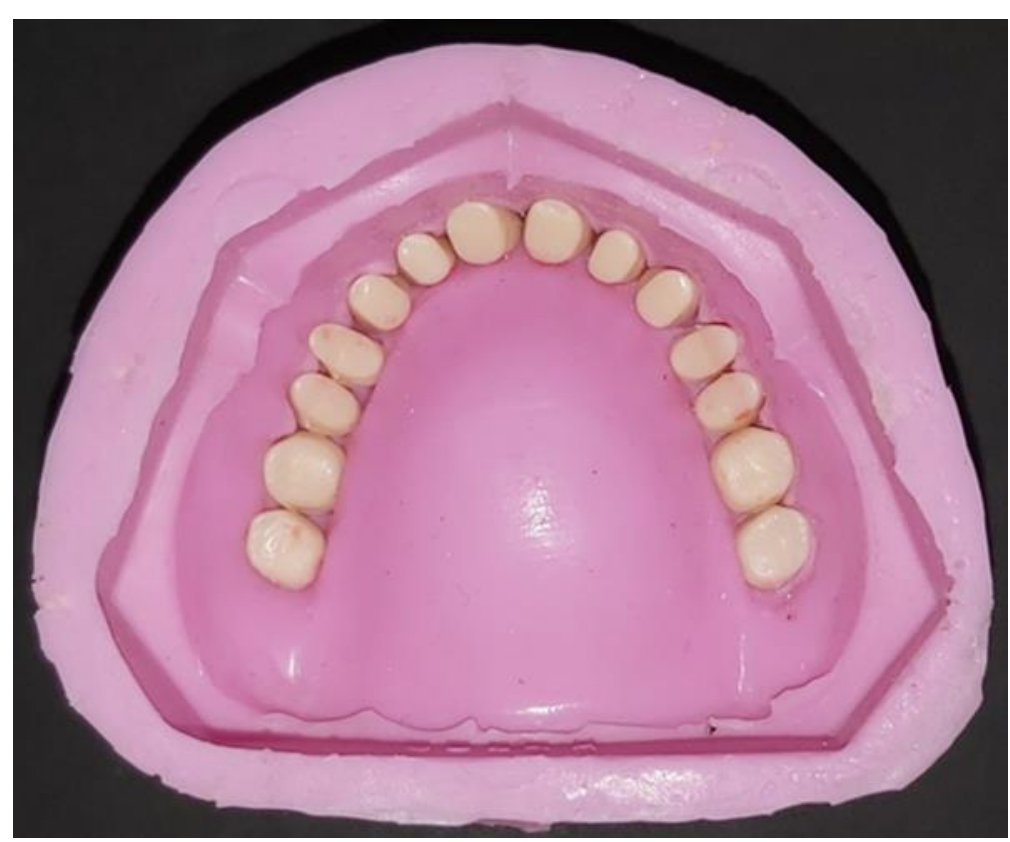

Figure 1: Setting of teeth inside elastic mold to fabricate dentures of similar design and thickness

\section{Fabrication of reinforcing meshes and insertion into final dentures:}

One of poured stone casts was scanned by optical scanner (3shape D850 desktop scanner 3Shape ${ }^{\circledR}$, Denmark) and digital data in STL format was imported into digital mesh design software -EXOCAD- (Autodesk San Rafael, CA, USA, partial module). The reinforcing mesh was designed to cover all palatal area and to extend to the crest of ridge, but not crossing it, anteriorly and bilaterally. The posterior part of edentulous ridge at tuberosity area was not covered rather a V-shaped notch was designed. The mesh was virtually relieved by $0.5 \mathrm{~mm}$ thickness wax. Mesh thickness at palatal vault and, on the ridge area was $0.7 \mathrm{~mm}$ and $0.9 \mathrm{~mm}$ respectively. The whole design process is shown in figure (2). The digital file of the designed mesh was then imported to SLA printer (FORMLAB, FORM2 USA) for the 
printing of fourteen reinforcing meshes in castable resin (Castable Flcablo 2, FORMLAB USA). Seven of the printed resin meshes were then sprued and invested for the fabrication of metal meshes. Phosphate-bonded, metal investing material (Ecovest PCS MTD COM) was mixed and poured into investing rings according to the manufacturer's instructions. Burnout of castable resin and casting of metal was then performed.
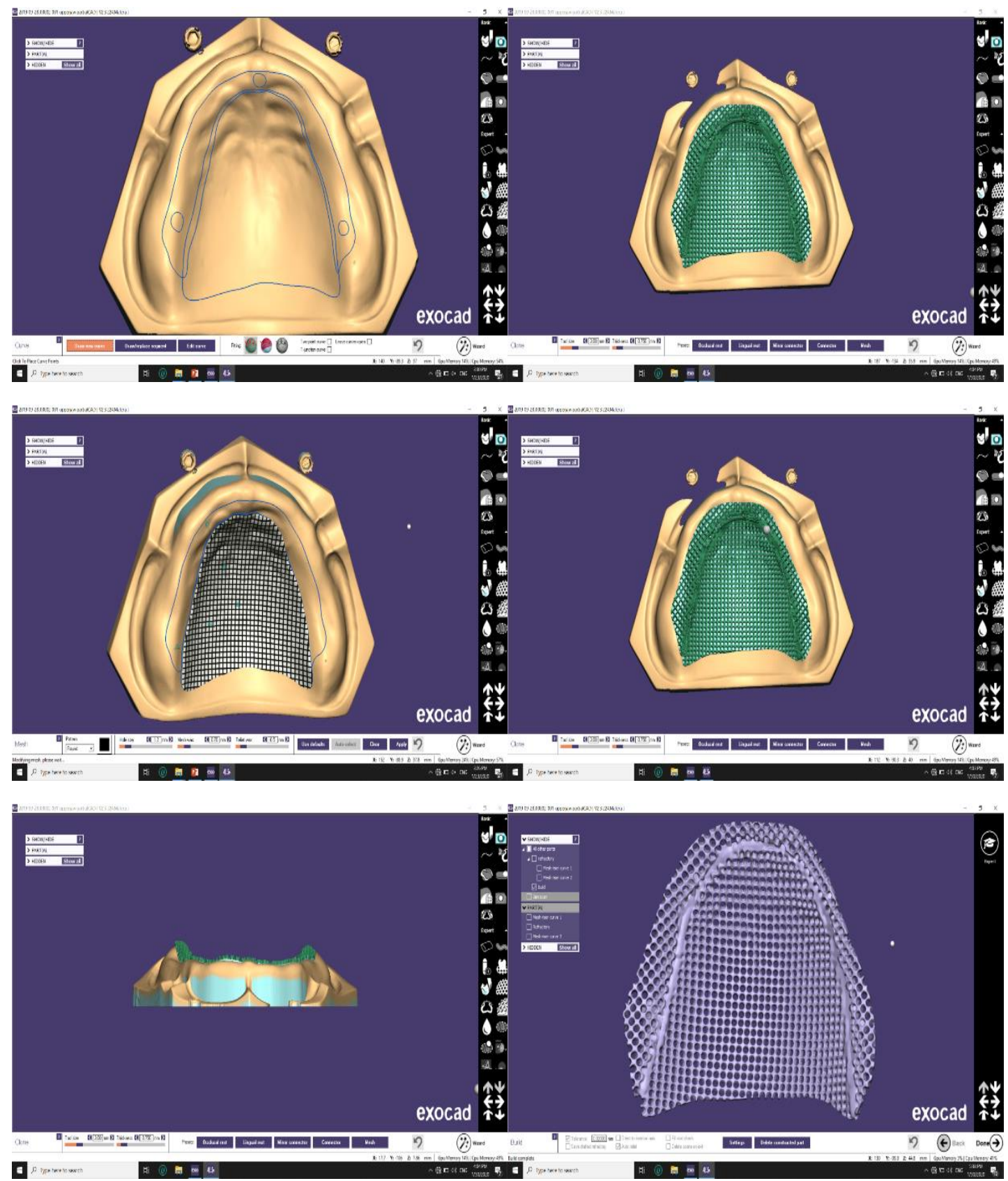

Figure 2: Digital planning of mesh framework

The same procedures were performed for the casting of PEEK meshes with only exception that after burn-out of the castable resin was performed, investing ring was pre-heated and PEEK was pressed as per for 2 press technique for pressing PEEK $^{22}$.

A temperature of $400^{\circ} \mathrm{C}$ was maintained for 90 minutes during the pressing of PEEK. Finally, the fabricated meshes were retrieved from investment material and finished. PEEK meshes were painted by VISIOLINK adhesive material (@ Bredent Chesterfield, UK) and light cured 
for 90 seconds to enhance the bond between PMMA and PEEK. The finished meshes were then kept in a small box lined with depron foam till the incorporation of meshes in dentures to be fabricated

The previously fabricated trial denture bases were flasked using the conventional method. After the flasking, wax elimination was performed, and dentures were constructed.

In group A: Heat-cured acrylic denture base material was mixed according to the manufacturer's instructions. Separating media was applied to the 2 halves of the flasks. The material was be packed in the dough stage. After trial packing, the cellophane paper was removed and before final closure, manufactured metal mesh was applied on the palatal area. Then final closure was done by applying pressure of $10,000 \mathrm{~N}$ in a hydraulic press. After bench curing and heat polymerization, the dentures were finished and polished. Similarly, additional six specimens were fabricated to produce all dentures of the control group.

In group B: The dentures were fabricated following the same procedures described for the control group except that PEEK reinforcing meshes were inserted in flask at the packing stage instead of metal ones.

After the fabrication of all the study samples, dentures were labeled by identification numbers, then submerged into saline for at least 24 hours before measuring the weight and fracture resistance.

\section{Testing of samples}

Before testing the fracture resistance, all the specimens were removed from the saline and weighted using a digital scale (Weigh Gram Barcelona - Spain). The results were recorded and tabulated. After that the specimens where loaded to evaluate the fracture resistance of the specimens.

Each specimen was horizontally mounted on a universal loading, computer-controlled testing machine (UTM, Lloyd IR 5k, Lloyd Industries LTD Hampshire, UK). The dentures were mounted with the occlusal surface oriented downward. A compressive load was applied at 90 degrees to each denture base through loading arm of machine on the tissue surface at the point where the midline of the denture crosses the line connecting the second premolars on each side. The downward load applied along the midline of the tissue surfaces of the denture was designed to be equivalent to the upward load on both sides. The speed of loading arm movement was $5 \mathrm{~mm} / \mathrm{s}$ and the load was gradually increased in magnitude till there was a sudden drop in load of more than $50 \%$ than the initial applied load as described by Polyzois ${ }^{23}$ At that point, the fracture resistance was recorded.

\section{Evaluation of fracture pattern:}

The fracture pattern of denture was also evaluated visually and classified based on the site and pattern of fracture as described by Choudhary ${ }^{24}$. The fracture was described as class I 
fracture if the fracture line passed through midline between central incisors and was extending posteriorly. Class II was defined if fracture line was running in a diagonal direction extending to the posterior extension and further away from midline. Moon-shaped fracture passing through labial or buccal flange without or without the separation of fractured segment was described as class III. Class IV was described as fracture line passing through dentoalveolar structure with loss of two or more teeth while in class $\mathrm{V}$ was considered as a fracture of a part of artificial tooth or separation of a single tooth from denture. One more class was added by the authors to the above-described classification, labelled as class VI, and described combined fracture at 2 or more sites of any of the previously mentioned locations.

\section{Statistical analysis:}

Statistical analysis was performed using IBM SPSS Statistics Version 2.0 for Windows. Data was presented as mean and standard deviation (SD). The significance level was set at $P \leq$ 0.05. Kolmogorov-Smirnov and Shapiro-Wilk tests were used to assess data normality.

Independent Student t-test was used to compare fracture resistance and weight of metalreinforced and PEEK-reinforced frameworks.

\section{RESULTS AND DISCUSSION}

\section{Fracture resistance:}

Values of maximum load in $\mathrm{N}$, stiffness in $\mathrm{N} / \mathrm{m}$ and work to rupture (fracture) in $\mathrm{J}$ after the loading of specimens were extracted from universal testing machine (UTM) and are presented in table: 1 .

The difference between the two groups was statistically significant $(P=0.004)$ where metalreinforced dentures withstood significantly higher maximum load before fracture compared to dentures in PEEK-reinforced group. Metal reinforced maxillary dentures could withstand maximum average load of $1686.63(\mathrm{~N})$ with corresponding value of $1160.22(\mathrm{~N})$ for PEEK reinforced maxillary denture group. The standard deviation (SD) value was higher in metal reinforced dentures $( \pm 325.06 \mathrm{~N})$ when compared with corresponding values in the PEEK reinforced group $( \pm 197.21 \mathrm{~N})$ which reflects the wider range of maximum load values in metal reinforced group.

Table 1: Mean \pm SD and P-value for the comparison of maximum load in $(N)$ between metal-reinforced and PEEK-reinforced frameworks

\begin{tabular}{lllc}
\hline & $\begin{array}{l}\text { Mean value of maximum } \\
\text { load (N) }\end{array}$ & $\begin{array}{l}\text { Standard deviation } \\
\text { (SD) }\end{array}$ & P-value \\
\hline Metal-reinforced framework & 1686.63 & \pm 325.06 & \\
PEEK-reinforced framework & 1160.22 & \pm 197.21 & $0.004^{*}$ \\
$*$ : Statistically significant at $P \leq 0.05$ & & \\
\hline
\end{tabular}




\section{Fracture pattern}

In PEEK-reinforced group, visual assessment of fracture lines revealed clear midline fracture extending posteriorly (class I) with complete separation of fractured parts in $57 \%$ of samples figure: 6 (d,e,f,g). Two samples representing 29\% showed class II fracture pattern with fracture line running in a diagonal direction extending posteriorly and further away from midline figure: 6 (a \& c). Only one specimen showed fracture line passing through dentoalveolar structure (class IV) which represents $14 \%$ of test group (Group b). Figure (6 b). In control group (group A), different fracture patterns were revealed; class (VI) was the most common pattern of fracture. A combination fracture presenting both half-moon shaped fracture, diagonal and midline fracture was observed in $57 \%$ of samples (as in figure: $5 \mathrm{a}, \mathrm{b}, \mathrm{c}$ \& g). Class II fracture pattern was found in $29 \%$ of specimens (Figure 5 e, f). Contrary to fractures observed in group B, there was an incomplete separation of fractured parts in most of control group specimens which showed peeling away of resin flacks from reinforcing metal framework.

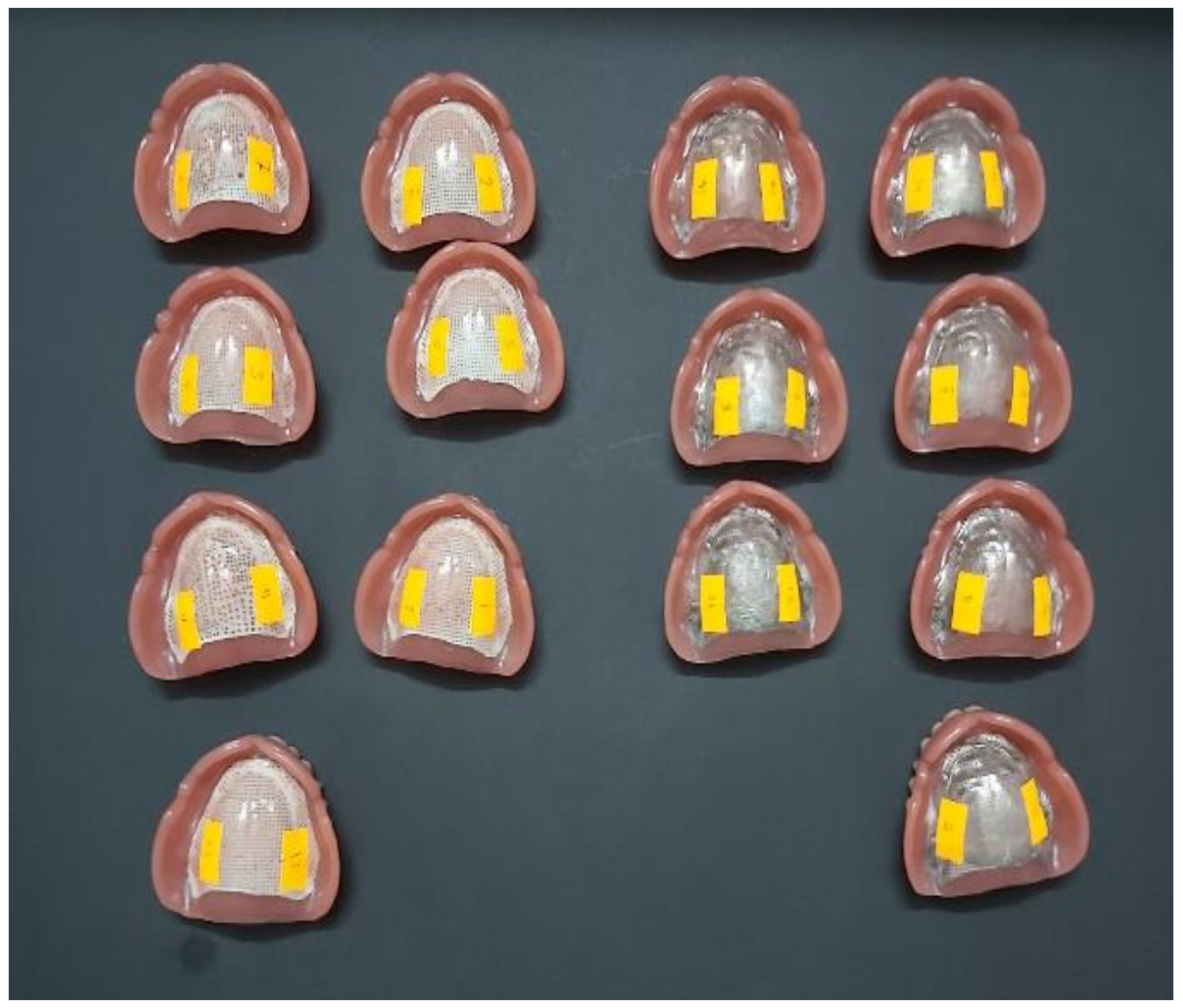

Figure 3: All samples collected and numbered before testing 


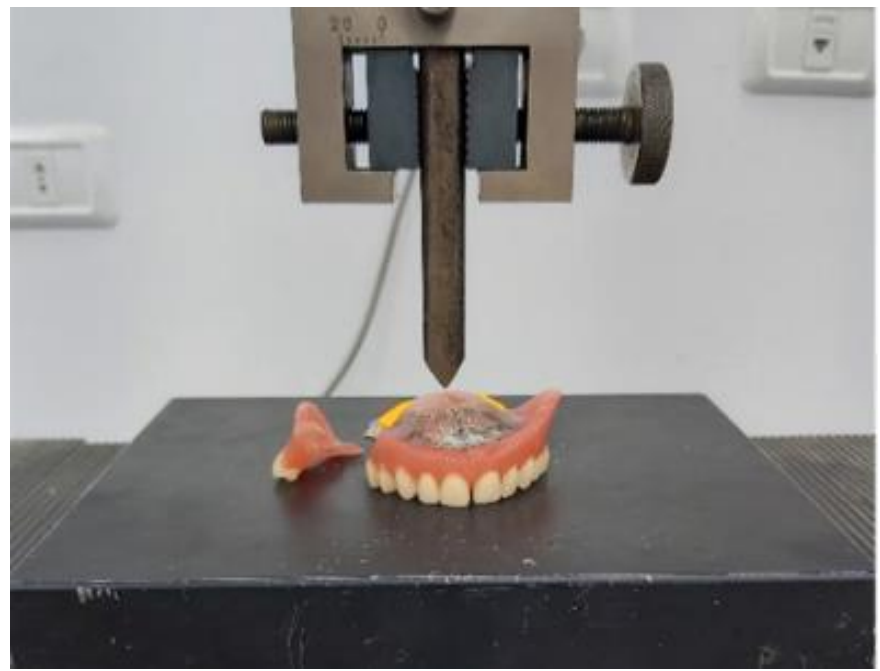

Figure 4 :Set up of test \& loading of samples
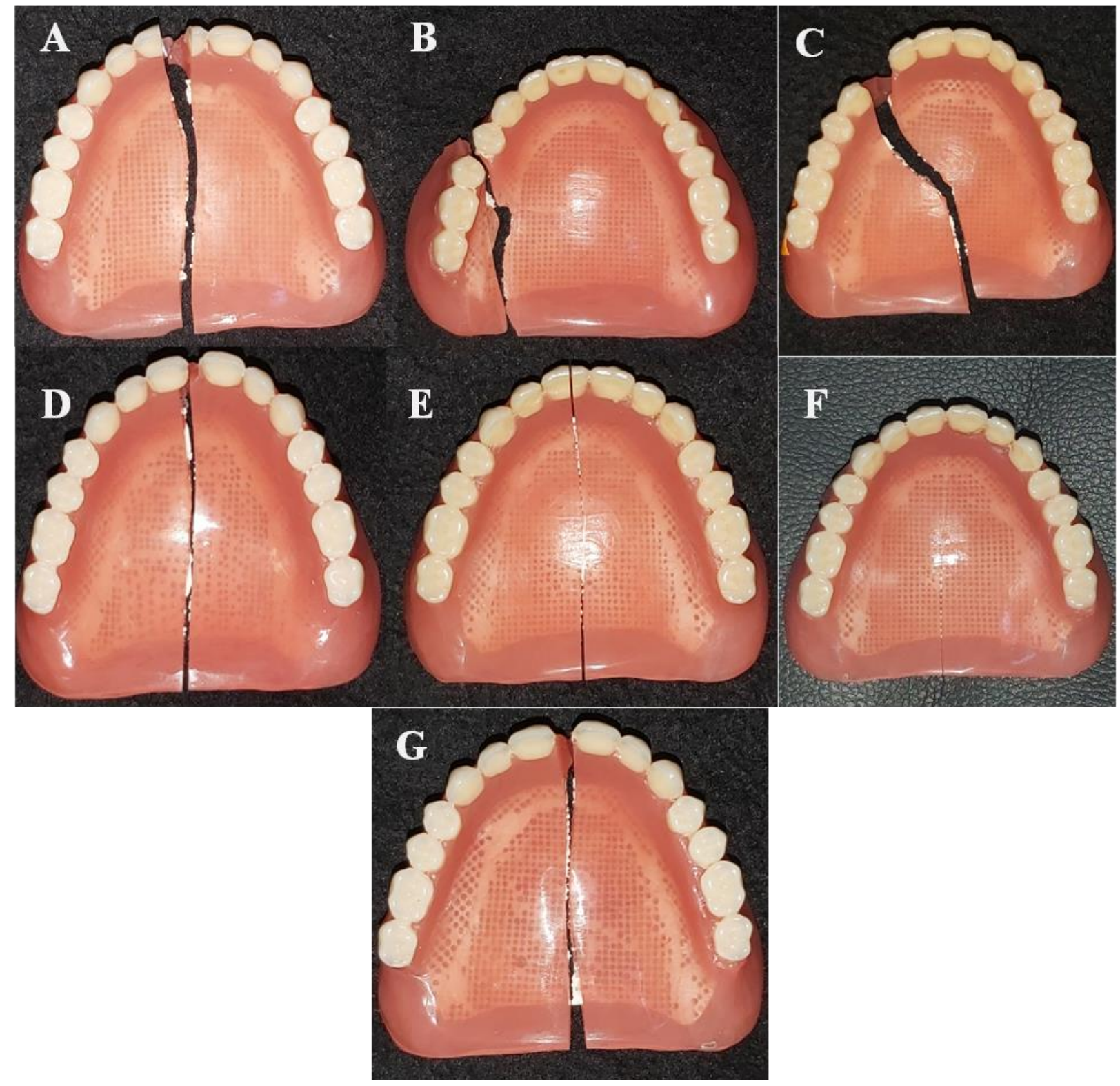

Figure 5: Fracture patterns of control group 


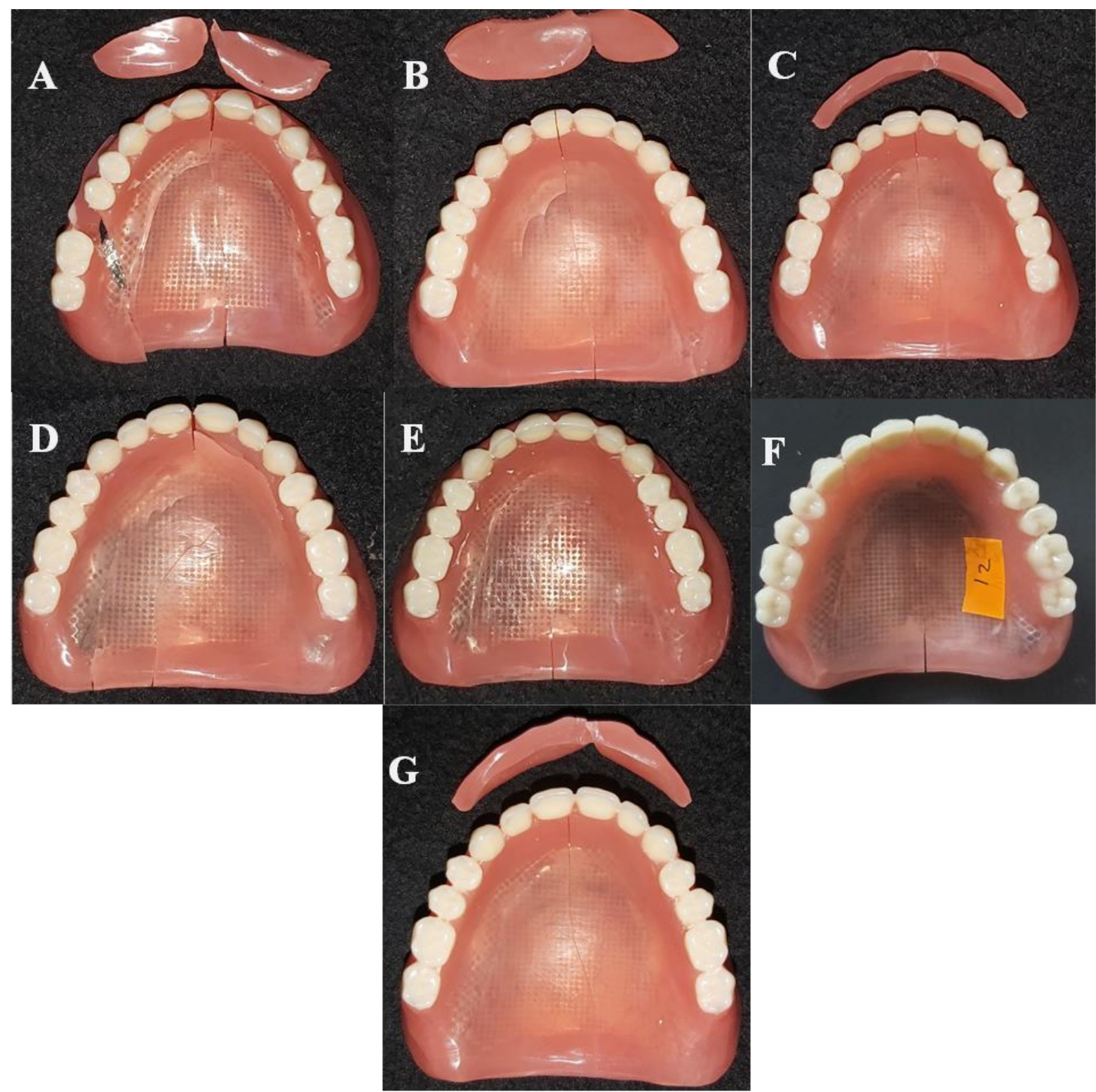

Figure 6: Fracture patterns of intervention group, class $I$ in $(D, E, F, G)$ class II in $(A \& C)$, class IV in (B).

\section{Weight measuring:}

Independent Student test (table: 2) showed that metal-reinforced framework displayed significantly higher mean weight values compared to PEEK-reinforced framework $(\mathrm{P}<0.001)$. The results revealed that using PEEK mesh decreased weight of denture by $26 \%$ compared to metal-reinforced denture.

Table 2: Mean \pm SD and P-value for the comparison of weight (g) between metal- and PEEK-reinforced frameworks.

\begin{tabular}{llll}
\hline & Metal-reinforced framework & PEEK-reinforced framework & P-value \\
\hline Weight $(\mathrm{g})$ & $34.44 \pm 0.65$ & $25.44 \pm 1.43$ & $<0.001^{*}$ \\
\hline
\end{tabular}

Conventional complete dentures fabricated using polymethyl methacrylate (PMMA) are considered as one of optimal treatment modalities for edentulous upper ridge. Unfortunately, the mechanical properties of this material are suboptimal where PMMA denture bases 
undergo fracture ${ }^{10}$. According to a survey performed by Darbar UR et $a^{25}{ }^{25}$, it was reported that $29 \%$ of all repairs were associated with midline fractures of complete dentures. Causes of such fracture pattern have been attributed to flexural failure caused by a combination of fatigue and stress concentration around fulcrum line due to differential resorption pattern in supporting bony areas between alveolar ridges and hard palate area specially in the region of median palatine raphe ${ }^{26}$. To overcome these deficiencies, many attempts have been made to enhance the mechanical properties of denture base acrylic amongst which is the use of reinforcing mesh made of higher mechanical properties material such as metal. The aim of the current study was to evaluate the fracture resistance of PEEK-reinforced maxillary complete denture bases and compare it with that of metal-reinforced bases.

The current study was designed as an in-vitro study as laboratory studies can be more easily controlled and standardized and as well yield accurate results especially when the experiments are concerned with comparative values ${ }^{21}$.

An educational maxillary "Nission model" was used to ensure an ideal condition of the ridge, and the palate. Model U-402 was selected as it represents limited undercuts and irregularities to avoid scratching of stone cast during repeated seating and removal of acrylic denture base during study procedures.

The elastomeric duplicate silicone material used was addition polysilicon material as it is considered one of the most dimensionally stable register materials. It also requires less time to recover the viscoelastic deformation compared to other rubber impression materials ${ }^{27}$. The silicone index was used as a mold to produce identical stone casts for standardization.

In our study, two different types of meshworks were used for the reinforcement of maxillary denture bases and were compared; the commonly used conventional metallic (Co-Cr) mesh and the newly introduced meshes made of PEEK material. A chromium cobalt alloy (Co 63.0 percent, Cr 29.5 percent, Mo 5.0 percent, Si 1.0 percent) was used for the casting of the metal frameworks because of its low cost, availability, and ease of manufacturing ${ }^{28}$. In a narrative review, Tekin, $S$ et al. ${ }^{29}$ reported that PEEK is used in dentistry as an alternative material to metal components in removable partial prostheses. PEEK as a polymer material eliminates metallic taste and allergic reactions which can be experienced with prosthetics metal components. Furthermore, the white color of PEEK can encourage it use as an alternative removable partial prosthesis material in areas of esthetic concern where display of metallic component would be unacceptable. In this study, Bio-HPP (High Performance Polymer), which is a PEEK variant was selected for the fabrication of PEEK frameworks. Bio-HPP is reinforced with special ceramic filler, which results in improved mechanical properties optimal for the use in the different dental fields including the field of prosthodontics. Muhsin SA. et al. ${ }^{30}$ found that flexural properties of PEEK reinforced denture base material was 
higher than that of non-reinforced PMMA and suggested the use of reinforced PEEK material as a higher strength denture base material in removable prosthesis construction. The grain size of ceramic fillers used for the reinforcement of PEEK ranges in size from 0.3 to $0.5 \mu \mathrm{m}$. Owing to this small grain size, homogeneity of material can be ensured, which is an important prerequisite for the outstanding material properties and consistent quality. The fine granularity of the filler particles as well provides the basis for the extremely good polishing properties that the material exhibits and minimal plaque adhesion ${ }^{31}$.

Digital workflow was selected for designing both types of meshwork frameworks to ensure the design standardization regarding the thickness and the extension of the mesh within the denture base material.

The 3Shape desktop scanner which was used in the current study is known for its accuracy and precision of scanning. It consists of LED, 2 x 5 MP cameras with an accuracy of $7 \mu \mathrm{m}$ (ISO) / $8 \mu \mathrm{m}$ (Implant bar) and a motion system supporting several axes for positioning the scanned object towards the light source and cameras. The light source projects well-defined lines onto the surface of the object, and the cameras acquire images of the lines ${ }^{32}$.

The mesh frameworks were designed to cover the hard-palatal area and to extend to the crest of the ridge but did not exceed the maximum curvature of the edentulous ridge to avoid unaesthetic metal display. Furthermore, half circular-shaped notches were designed on maxillary tuberosity anatomical area to avoid any increase in thickness of that area that may interfere with occlusion in case of actual clinical situation. The mesh was virtually relieved by $0.5 \mathrm{~mm}$ thickness of wax to create a space for incorporation of acrylic to directly fit over oral mucosa to accept future relining.

In addition to being commercially available, Formlabs printing system (printer and castable resin) is SLA-based printing system. Such a printing system provides accurate and repeatable castable patterns. According to the manufacturer's instructions, the printed framework was rinsed twice in $96 \%$ ethanol solution in an ultrasonic bath to remove excess uncured material. Following the ultrasonic bath, a maximum of 5-minute alcohol rinse was performed, any excessive rinsing was avoided as it could lead to surface defects in the printed framework ${ }^{33}$. For the standardization of experiment set-up, for2press fabrication and conventional casting techniques were selected for the fabrication of PEEK and metal mesh frameworks respectively. Those two techniques are somewhat similar in technical steps except for the type of material and cycles used.

All dentures were stored in $37^{\circ} \mathrm{C}$ water for 24 hours before the testing procedures to eliminate the influence of PMMA water resorption on mechanical properties of all dentures. The fracture resistance was measured using a universal testing machine at a crosshead speed of $5.0 \mathrm{~mm} /$ minute. A compressive load was applied to each specimen in a downward direction 
along the midline of the tissue surfaces of the denture bases to be equivalent to the upward load on both sides of bases combined with unyielding support in the center of the plate, thus simulating the actual clinical scenario ${ }^{31}$.

The flexural strength of denture base materials is generally evaluated using a three-point bending test on a beam-shaped sample according to the British standard BS 20795-1:2013, and ISO standard number: $20795^{34}$. Nevertheless, in this study to mimic the actual clinical situation, denture-shaped specimens were used and the maximum load the denture baseframework complex could withstand was obtained and was considered as a direct indicator of the fracture resistance property of the material rather than using the above-mentioned standardized tests ${ }^{35}$.

The results revealed that the maximum load the specimens could withstand in the control group (group A) were significantly higher than the specimens in test group (group B). These results are coinciding with the reported data in the literature where the maximum load values for the PEEK and Co-Cr were $150 \mathrm{MPa}$ and $464 \pm 70 \mathrm{MPa}$; respectively ${ }^{36},{ }^{37}$. Zoidis P. et al 38 suggested preforming some design modifications in PEEK frameworks. These changes involve the increase in the thickness of framework material to improve the mechanical performance of material as reinforcing mesh framework. Similarly, Dawson JH et al. 39 reported better mechanical properties of PEEK frameworks compared to metal ones when used in larger thickness than metal for both upper CDs and lower implant supported fixed dentures.

The low specific gravity of PEEK material $\left(1.32 \mathrm{~g} / \mathrm{cm}^{3}\right)$ can safely permit the modification of framework design through the increase in the thickness of the mesh without the fear of increasing the weight of prosthesis ${ }^{40}$. This is in accordance with the findings of the current study where metal-reinforced dentures displayed significantly higher mean weight values compared to their PEEK-reinforced counterparts $(P<0.001)$. Our results revealed that using PEEK mesh decreased weight of dentures by $26 \%$ compared to metal-reinforced dentures. Furthermore, the lighter weight of PEEK-reinforced dentures can positively affect the retention of maxillary complete denture and consequently the patient's satisfaction. CostaPalau et al. ${ }^{41}$ used peek for the fabrication of maxillofacial prostheses and reported satisfactory results by the patients. In the same context, Zoidis P. et al. ${ }^{31}$ evaluated patients' satisfaction when using PEEK frameworks compared to metal ones in RPDs and as well measured the weight of prosthesis. The findings of the later study showed that PEEK RPDs are lighter in weight compared to metal ones due to the difference in specific gravity between the two materials. The patients' acceptance of PEEK RPDs was also higher than that of the patients in the metal group. The difference in weight between the two groups $(27.5 \%)$ in 
study by Zoidis $P$. et al. ${ }^{38}$ was almost similar to the weight difference in our study (26\%) between the metal and PEEK group.

Therefore, it can be suggested that for the sake of future studies increasing the thickness of PEEK framework should be evaluated in relation to improvement of its mechanical properties and reinforcing qualities as a denture framework material. However, caution should be exercised that such an increase in thickness of framework material be performed within physiologic limits that would not interfere with the speech of patients. As per the literature, maximum thickness of palatal area of maxillary complete denture can be increased up to 3.75 mm. which would be maximum range of increase in PEEK framework for future studies ${ }^{42}$.

Nazari $V$. et al. 43divided the fracture mode of composite prosthetic devices which consist of 2 or more materials into three patterns; a fracture which initially occur in the surface material and propagate to the framework, the second pattern is a fracture that occur at the interface of reinforcing framework and bulk material, and a third pattern that represents a mixed pattern of fracture with a combination of different fracture patterns at different sites. By gross inspection of fracture patterns of our study samples, a clear midline fracture of both acrylic base material and reinforcing PEEK framework with limited or no tags and complete separation of fractured parts was observed in group B (test group). This fracture pattern indicates the presence of true interlocking (bonding) between the PEEK and resin base material that the fracture did not propagate through the interface between the 2 materials ${ }^{44}$. Thus, it can be suggested that for increasing mechanical properties of composite prosthesis (denture and reinforcing framework), the thickness of mesh can be increased which increases the overall fracture resistance of whole prosthesis. ${ }^{24}$ Most of test group fractures were of class $I$ or class II types (midline and para midline fracture patterns). On the other hand, different fracture patterns were observed in control group where class VI type of fracture was predominantly observed which combined midline of denture base and half-moon fracture passing through labial or buccal flange. Contrary to the fracture pattern of test group, the control group showed incomplete separation of fractured parts and peeling of some resin flacks from metal framework which indicates that there was no true bond between the reinforcing metal framework and resin base material. Therefore, the crack propagated through the weakest phase of composite prosthesis which is represented by the interface between metal and PMMA and resulted in the peeling of PMMA from metal framework. The finding of our study is in accordance with the results of Sang-Hui Yu et al. ${ }^{45}$ who revealed incomplete fracture pattern which occurred only in the resin base material and propagated through the interface between resin base and reinforcing metal framework resulting in the peeling of PMMA when evaluating the fracture load and toughness of metal-reinforced dentures. 


\section{CONCLUSION}

Based on the findings of the current study, it can be concluded that using PEEK mesh frameworks with thickness of 0.7 to $0.9 \mathrm{~mm}$ did not improve the fracture strength of conventional maxillary complete dentures. The fracture strength of PEEK-reinforced dentures was significantly lower than that of metal-reinforced counterparts with the same thickness. However, the light weight of the material can allow for the increased thickness of framework and consequently improved mechanical properties of PEEK as a reinforcing material and with no negative influence on the retention or esthetic of the prosthesis. Nevertheless, before such an assumption can be made, there is a need for future, well designed studies to validate or refute such assumption and enable evidence-based recommendations.

\section{REFERENCES:}

1. Cunha, T. R. et al. A randomised trial on simplified and conventional methods for complete denture fabrication: Masticatory performance and ability. J. Dent. 41, 133142 (2013).

2. Sheng, T. J., Shafee, M. F., Ariffin, Z. \& Jaafar, M. Review on poly-methyl methacrylate as denture base materials. Malaysian J. Microsc. 14, 1-16 (2018).

3. Bhandari, S. Outcome of single maxillary complete dentures opposing mandibular teeth: A need to introspect on the prosthodontic treatment protocol. (2016) doi:10.4103/0972-4052.167941.

4. K K Narva , P K Vallittu, H Helenius, A. Y.-U. Clinical Survey of Acrylic Resin. 14, 219-224 (2001).

5. IM, S.-M., Huh, Y.-H., Cho, L.-R. \& Park, C.-J. Comparison of the fracture resistances of glass fiber mesh- and metal mesh-reinforced maxillary complete denture under dynamic fatigue loading. J. Adv. Prosthodont. 9, 22 (2017).

6. Parvizi, A. et al. BASIC SCIENCE RESEARCH Comparison of the Dimensional Accuracy of Injection-Molded Denture Base Materials to that of Conventional Pressure-Pack Acrylic Resin Comparison of Dimensional Accuracy r Parvizi et al. J. Prosthodont. 13, 83-89 (2004).

7. Alla, R. K., Swamy, K. N., Vyas, R. \& Konakanchi, A. Conventional and Contemporary polymers for the fabrication of denture prosthesis: part I - Overview, composition and properties. Int. J. Appl. Dent. Sci. 1, 82-89 (2015).

8. Vipul Somani, M., Khandelwal, M., Punia, V. \& Sharma, V. The effect of incorporating various reinforcement materials on flexural strength and impact strength of polymethylmethacrylate: A meta-analysis. (2019) doi:10.4103/jips.jips_313_18.

9. Doğan, O. M. et al. The effect of esthetic fibers on impact resistance of a conventional 
heat-cured denture base resin. Dent. Mater. J. 26, 232-239 (2007).

10. Vallittu, P. K. \& Lassila, V. P. Reinforcement of acrylic resin denture base material with metal or fibre strengtheners. J. Oral Rehabil. 19, 225-230 (1992).

11. Carroll, C. E. \& von Fraunhofer, J. A. Wire reinforcement of acrylic resin prostheses. J. Prosthet. Dent. 52, 639-641 (1984).

12. Chen, S. Y., Liang, W. M. \& Yen, P. S. Reinforcement of acrylic denture base resin by incorporation of various fibers. J. Biomed. Mater. Res. 58, 203-208 (2001).

13. Shimizu, H., Mori, N. \& Takahashi, Y. Use of metal conditioner on reinforcement wires to improve denture repair strengths. N. Y. State Dent. J. 74, 26-28 (2008).

14. Morrow, R. M., Reiner, P. R., Feldmann, E. E. \& Rudd, K. D. Metal reinforced silicone-lined dentures. J. Prosthet. Dent. 19, 219-229 (1968).

15. Berry, H. H. \& Funk, O. J. Vitallium strengthener to prevent lower denture breakage. J. Prosthet. Dent. 26, 532-536 (1971).

16. Kawano, F., Miyamoto, M., Tada, N. \& Matsumoto, N. Reinforcement of acrylic resin denture base with a Ni-Cr alloy plate. Int. J. Prosthodont. 3, 484-488 (1990).

17. el Ghazali, S., Glantz, P. O., Strandman, E. \& Randow, K. On the clinical deformation of maxillary complete dentures. Influence of denture-base design and shape of denture-bearing tissue. Acta Odontol. Scand. 47, 69-76 (1989).

18. Burns, D. R. The mandibular complete overdenture. Dent. Clin. North Am. 48, 603623 (2004).

19. George Zarb John Hobkirk Steven Eckert Rhonda Jacob. Prosthodontic Treatment for Edentulous Patients. (Elsevier, 1990).

20. Shimizu, H. et al. Fracture strength of metal-based complete maxillary dentures with a newly designed metal framework. Int Chin J Dent 5, 35-38 (2005).

21. Bathala, L., Majeti, V., Rachuri, N., Singh, N. \& Gedela, S. The Role of Polyether Ether Ketone (Peek) in Dentistry-A Review. J. Med. Life 12, 5-9.

22. Emam, N. S., el-deen, F. S. \& Abdel-salam, S. M. Prevelance Of Oral And Respiratory Microorganisms On The Cad-Cam Versus Press Form Of Poly-EtherEther Ketone Material For Oral And Maxillofacial Prosthesis. Alexandria Dent. J. 44, 38-44 (2019).

23. Polyzois, G. L., Andreopoulos, A. G. \& Lagouvardos, P. E. Acrylic resin denture repair with adhesive resin and metal wires: Effects on strength parameters. J. Prosthet. Dent. 75, 381-387 (1996).

24. Choudhary, S. Complete denture fracture-A proposed classification system and its incidence in National Capital Region population: A survey. (2019) doi:10.4103/jips.jips_312_18. 
25. Darbar, U. R., Huggett, R. \& Harrison, A. Denture fracture--a survey. Br. Dent. J. 176, 342-345 (1994).

26. H. Causes and types of complete denture fracture. Zanco J. Med. Sci. (Zanco J Med Sci) 15, (2018).

27. Lindquist, T. J., Narhi, T. O. \& Ettinger, R. L. Denture duplication technique with alternative materials. J. Prosthet. Dent. 77, 97-98 (1997).

28. Becker, C. M., Kaiser, D. A. \& Goldfogel, M. H. Evolution of removable partial denture design. J. Prosthodont. Off. J. Am. Coll. Prosthodont. 3, 158-166 (1994).

29. Tekin, S., Cangül, S., Adıgüzel, Ö. \& Değer, Y. Areas for use of PEEK material in dentistry. Int. Dent. Res. 8, (2018).

30. Muhsin, S., Wood, D., Hatton, P., Johnson, A. \& Sereno, N. The Effect of Processing Conditions on the Flexural Strength of Polyetheretherketone (PEEK) Used as Innovative Denture Base Material. (2015).

31. Zoidis, P., Papathanasiou, I. \& Polyzois, G. The Use of a Modified Poly-Ether-EtherKetone (PEEK) as an Alternative Framework Material for Removable Dental Prostheses. A Clinical Report. J. Prosthodont. 25, 580-584 (2016).

32. Sousa, M. V. S., Vasconcelos, E. C., Janson, G., Garib, D. \& Pinzan, A. Accuracy and reproducibility of 3-dimensional digital model measurements. Am. J. Orthod. Dentofac. Orthop. Off. Publ. Am. Assoc. Orthod. its Const. Soc. Am. Board Orthod. 142, 269-273 (2012).

33. Zhang, Z.-C., Li, P.-L., Chu, F.-T. \& Shen, G. Influence of the three-dimensional printing technique and printing layer thickness on model accuracy. J. Orofac. Orthop. $=$ Fortschritte der Kieferorthopadie Organ/official J. Dtsch. Gesellschaft fur Kieferorthopadie 80, 194-204 (2019).

34. (BSI), B. S. I. British Standards Institution. Dentistry Base Polymers. Part 1: De, 20795-1:2013;

35. Zidan, S. et al. materials Evaluation of Equivalent Flexural Strength for Complete Removable Dentures Made of Zirconia-Impregnated PMMA Nanocomposites. Materials (Basel). 13, (2020).

36. Rocha, R., Pinheiro, A. L. B. \& Villaverde, A. B. Flexural strength of pure Ti, Ni-Cr and Co-Cr alloys submitted to Nd:YAG laser or TIG welding. Braz. Dent. J. 17, 2023 (2006).

37. Bechir, E. et al. The Advantages of BioHPP Polymer as Superstructure Material in Oral Implantology. Mater. Plast. 53, (2016).

38. Zoidis, P., Papathanasiou, I. \& Polyzois, G. The Use of a Modified Poly-Ether-EtherKetone (PEEK) as an Alternative Framework Material for Removable Dental 
Prostheses. A Clinical Report. 13, (2015).

39. Dawson, J. H., Hyde, B., Hurst, M., Harris, B. T. \& Lin, W.-S. Polyetherketoneketone (PEKK), a framework material for complete fixed and removable dental prostheses: A clinical report. J. Prosthet. Dent. 119, 867-872 (2018).

40. Kurtz, S. M. Chapter 1 - An Overview of PEEK Biomaterials. in PEEK Biomaterials Handbook (Second Edition) (ed. Kurtz, S. M.) 3-9 (William Andrew Publishing, 2019). doi:https://doi.org/10.1016/B978-0-12-812524-3.00001-6.

41. Costa-Palau, S., Torrents-Nicolas, J., Brufau-de Barberà, M. \& Cabratosa-Termes, J. Use of polyetheretherketone in the fabrication of a maxillary obturator prosthesis: a clinical report. J. Prosthet. Dent. 112, 680-682 (2014).

42. Mazaro, J. V. Q. et al. Influence of different base thicknesses on maxillary complete denture processing: linear and angular graphic analysis on the movement of artificial teeth. J. Craniofac. Surg. 22, 1661-1665 (2011).

43. Nazari, V., Ghodsi, S., Alikhasi, M., Sahebi, M. \& Shamshiri, A. R. Fracture Strength of Three-Unit Implant Supported Fixed Partial Dentures with Excessive Crown Height Fabricated from Different Materials. J. Dent. (Tehran). 13, 400-406 (2016).

44. Awaja, F., Zhang, S., Tripathi, M., Nikiforov, A. \& Pugno, N. Cracks, microcracks and fracture in polymer structures: Formation, detection, autonomic repair. Prog. Mater. Sci. 83, 536-573 (2016).

45. Yu, S.-H., Oh, S., Cho, H.-W. \& Bae, J.-M. Reinforcing effect of glass-fiber mesh on complete dentures in a test model with a simulated oral mucosa. J. Prosthet. Dent. 118, 650-657 (2017).

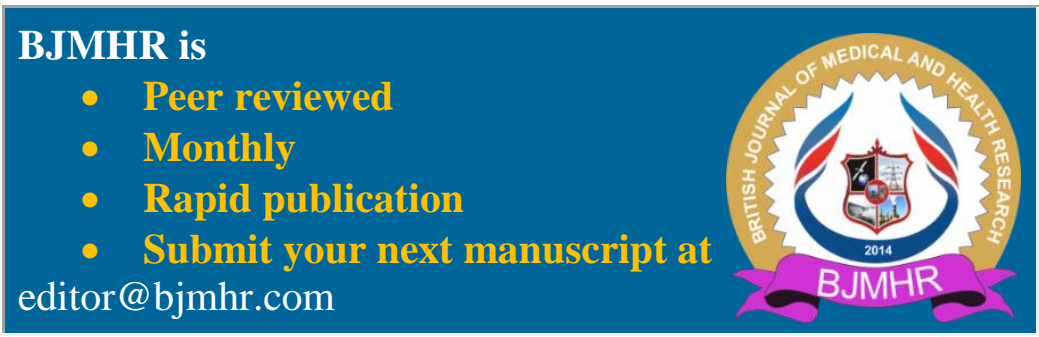

